\title{
Two-dimensional stick-slip on a soft elastic polymer: Pattern generation using atomic force microscopy
}

\author{
J A Watson ${ }^{1,3}$, C L Brown ${ }^{1}$, S Myhra ${ }^{1,2}$, G S Watson ${ }^{1}$ \\ ${ }^{1}$ Nanoscale Science and Technology Centre, School of Science, Griffith University, Kessels Rd, Nathan, \\ 4111, Qld, Australia; \\ ${ }^{2}$ Department of Materials, University of Oxford, Begbroke Science Park, Sandy Lane, Yarnton OX5 1PF, \\ UK
}

\begin{abstract}
It has been demonstrated that it is possible to create laterally differentiated frictional patterning and threedimensional structures using the Atomic Force Microscope (AFM) probe on the surface of a soft elastic polymer, poly(dimethylsiloxane) (PDMS). The resulting effect of contact mode imaging at low loading forces $(<100 \mathrm{nN})$, observed in the lateral force mode revealed a homogeneous pattern on the PDMS surface exhibiting higher friction. With higher loading forces $(>>100 \mathrm{nN})$ the effect is non-uniform resulting in structures with depths on the nanometre scale. The topographic and frictional data revealed stick-slip responses in both the fast (orthogonal to the long axis of the lever) and slow (parallel to the long axis of the lever) directions of probe travel from scanning in a raster pattern. The stick-slip events manifest in the form of a series of shallow channels spaced evenly apart on the polymer surface. Detailed friction loop analysis acquired during the manipulation process showed that the lateral force changed according to the strength of trapping of the tip with the polymer surface exhibiting significant inplane deformation due to lateral forces being imposed. An incremented increase in the initial loading force resulted in an increase in in-plane displacement and a greater spacing between the stick lines/channels in the slow scan direction. A decrease in channel length in the fast scan direction is also observed as a result of an increase in static friction with normal force, resulting in greater surface deformation and shorter track length for sliding friction.
\end{abstract}

PACS: 68.37.Ps, 81.65.Cf and 82.35.Lr

\footnotetext{
${ }^{3}$ Author to whom correspondence should be addressed: Jolanta.Watson@Griffith.edu.au.
} 


\section{Introduction}

Precise manipulation leading to laterally differentiated regions on polymeric materials is required for a range of applications/devices including confinement and separation of bio-species and array structures for multi-channel/multi-analyte biosensors. Likewise, it has been found that patterning of surfaces will hinder or promote adhesion of various chemistries/(bio)chemicals (Blach-Watson et al 2004, Nicolau et al 1999). While purposeful manipulation is currently taking place routinely on the $\mu \mathrm{m}$-scale, it is now apparent that processes and devices will be evolving into the nm-regime. Micro-machining is now a wellestablished family of technologies - e.g. directional chemical etching, preferential sputtering by focused ion beams, removal of material by laser ablation, micro-abrasion and other micro-scale mechanical wear mechanisms. More recently Scanning Probe Microscopy (SPM), and particularly the adjunct, Atomic Force Microscopy (AFM), have become another addition to the variety of tools available. While some of the technologies rest on a firm scientific basis, i.e. chemical etching and ion beam sputtering, the underlying science is much less satisfactory in the case of mechanical manipulation by SPM methods. At present the explanatory models for tip-induced manipulation of polymer surfaces are in their infancy. Earlier studies have approached the problem from several perspectives, generally based on insight gained from unrelated materials (Hector et al 1998, Schmid et al 1998, Fang et al 2000, Bhushan 2001, Pickering and Vancso 1999, Capella et al 2002). A few studies (e.g., Elkaakour et al 1994, Haugstad et al 1998, Schmidt et al 1999, 2003a, 2003b) have investigated polymer materials scanned over reasonably small scan ranges $(\leq 5 \times 5 \mu \mathrm{m})$ with results showing the formation of polymer bundles (polymer material being collected into linear bundles (or clumps) parallel to the long axis of the lever) as a result of action by the AFM probe.

The principal objective in this paper is to develop an understanding of tip-induced manipulation of a relatively soft elastic polymer with a focus on the directional response of the AFM probe during manipulation. Poly(dimethylsiloxane), (PDMS), has been chosen as a model polymer as it is now routinely used in a wide range of patterning and bio-chemical isolation and biomedical applications (Xia et al 1998, Kane et al 1999, Brinkmann et al 2001, Schnyder et al 2003, Ikada 1994), and has a multitude of potential applications including insulation and anti-fouling coatings (Kim et al 1999, Hillborg and Gedde 1999, Pike et al 1996). It offers a range of useful physical, mechanical and chemical properties including transparency, surface hydrophobicity, constant and high ductility over a wide range of temperatures, low toxicity, high electrical resistance, long-term stability and flexibility (Olah et al 2005). Of particular relevance to this study is the use of PDMS in soft lithography. The fabrication and use of micro/nano stamps and fluidic channels using PDMS material has been demonstrated in a number of studies (e.g., Kane et al 1999, Hu et al 1999, Schueller et al 1999, Deng et al 2002). An investigation of 
tribological properties and the lithographic outcomes on PDMS surfaces via AFM manipulation will contribute greatly toward an understanding of responses by soft polymeric materials.

\section{Experimental Section}

\subsection{Specimen materials}

PDMS (Sylgard ${ }^{\circledR}$-184) was supplied by Dow Corning as a two part silicone elastomer. The base and curing agent were mixed at a 10:1 weight ratio, spin-coated onto atomically flat silicon wafer substrates and left to cure in an ambient environment $\left(25^{\circ} \mathrm{C}\right.$ and $55 \%$ relative humidity) for 48 hours prior to any analysis or manipulation. The average thickness of the polymer was ca. $3 \mu \mathrm{m}$ determined using a profilometer (Tencor Alpha-Step 200) and the ThermoMicroscope Explorer. Typical properties of the polymer are presented in table 1 below.

Table 1. PDMS properties.

\begin{tabular}{ll}
\hline PROPERTY & CHARACTERISTICS \\
\cline { 1 - 2 } Physical & \\
Appearance & Transparent, optical detection $240-1100 \mathrm{~nm}$ (McDonald and \\
& Whitesides 2002, Dow Corning) \\
Young's Modulus & Tunable, typically $750-1700 \mathrm{kPa}$ (Armani et al 1998, Unger \\
Thermal Conductivity & et al 2000, Roca-Cusachs et al 2005) \\
Coefficient of thermal expansion & $0.2 \mathrm{~W} /(\mathrm{m} \cdot \mathrm{K})$ (Dow Corning) \\
Surface Free Energy & $310 \mu \mathrm{m} /\left(\mathrm{m} \cdot{ }^{\circ} \mathrm{C}\right)$ (Dow Corning) \\
\hline Electrical & Low; $\sim 20 \mathrm{erg} \cdot \mathrm{cm}^{2}$ (Chaudhury and Whitesides 1991) \\
\hline Breakdown voltage & $2 \times 10^{7} \mathrm{Vm}^{-1}$ (Dow Corning) \\
\hline
\end{tabular}

\subsection{AFM instrumentation}

The work was carried out on multi-technique/multi-mode Scanning Probe Microscope (SPM) instruments: a ThermoMicroscope TMX-2000 Explorer and Discoverer. The Discoverer was utilized for section 3.2 only, 'frictional pattern generation', all other work was performed using the Explorer. The instruments are based on the detection of tip-to-surface forces through the monitoring of the optical deflection of a laser beam incident on a force-sensing/imposing lever. Scanning with the Explorer is instigated by probe/lever motion with the stage assembly remaining stationary unlike the Discoverer where the stage assembly instigates the scanning motion. A Discoverer $70 \times 70 \mu \mathrm{m}^{2}$ and Explorer $130 \times 130$ $\mu \mathrm{m}^{2}$ tripod scanner, with a z-range of $9.7 \mu \mathrm{m}$, was used in order to cover the scales of lateral and topographical differentiation; the fields-of-view ranged from 70×70 (Discoverer) and 130×130 (Explorer) down to $2 \times 2 \mu \mathrm{m}^{2}$. The analyses were carried out under air-ambient conditions $\left(20-23^{\circ} \mathrm{C}\right.$ and $60-70 \%$ relative humidity). 


\subsection{Probe}

The probe consisted of a lever with an integral conical tip attached at its free end. The tip-to-surface point of contact defines the interaction volume, of molecular to micron size, from which information is extracted, (e.g., topography, strength of in-plane and out-of-plane forces, etc.) and through which purposeful manipulation is effected. In the context of AFM analysis many polymers can be considered as 'soft' objects; imposition of forces at the point of contact will cause deformation and indentation, and will lead to an increase in contact area. Accordingly it is necessary to use levers with low force constants (e.g., $k_{N} \leq 0.1 \mathrm{Nm}^{-1}$ ) in order to improve resolution and avoid unintended surface modification. Levers with higher force constants (e.g., $k_{N} \geq 4 \mathrm{Nm}^{-1}$ ) will allow surface manipulation to occur.

The characteristics of probes employed in the present study are listed in table 2. Beam-shaped levers (Ultrasharp NT-MDT) were used throughout the project in order to ensure that only simple bending modes needed to be considered. The data for radius of curvature at the tip apex, $R_{\text {Tip }}$, aspect ratio of the tip, $A_{r}$, and surface chemistry are summarized from the suppliers' specifications. The values of the force constant for normal deflection, $k_{N}$, of individual probes were determined from the resonance method described by Cleveland et al (1993), and the torsional, $k_{T}$, and longitudinal, $k_{L}$, force constants were calculated from the standard expressions for a long and thin lever, described by Gibson et al (1997).

Table 2. Probe specifications. Values stated by the manufacturer of the radius of curvature at tip apex, $A_{r}$, $<10 \mathrm{~nm}$, with the opening half-angle of the tip $<10^{\circ}$. Surface chemistry of the tip was determined by the native Si-oxide film, (subject to the usual caveat about the likely presence of hydrocarbon contamination). $k_{N}, k_{L}$ and $k_{T}$ are the force constants of the lever for normal, longitudinal and torsional deformation, respectively.

\begin{tabular}{cccc}
\hline Probe & $\boldsymbol{k}_{\boldsymbol{N}}\left(\mathrm{Nm}^{-1}\right)$ & $\boldsymbol{k}_{\boldsymbol{T}}\left(\mathrm{Nm}^{-1}\right)$ & $\boldsymbol{k}_{\boldsymbol{L}}\left(\mathrm{Nm}^{-1}\right)$ \\
\hline A & 0.035 & 7.2 & 4.7 \\
B & 6.8 & 150 & 95 \\
C & 1.98 & 27 & 17 \\
D & 7.1 & 155 & 100 \\
E & 0.1 & 15 & 10 \\
F & 10.3 & 220 & 145 \\
G & 0.07 & 11 & 7.4 \\
H & 14 & 300 & 200 \\
\hline
\end{tabular}

\subsection{Imaging and Manipulation}

Under normal topographical imaging conditions, a characteristic of most SPM instruments is an 'overscan' along the fast scan direction (i.e., perpendicular to the long axis of the lever). The overscan is generated by the instrument scanning beyond a specified field of view (typically ca. 25\%). Most 
instrumental software is programmed to omit (or cut out) this overscan prior to the formation of a computer generated topographical image. As a result, this overscan is generally ignored. This overscan feature is intended as a means of removing the static friction feature from images. Thus for $20 \times 20$ and $10 \times 10 \mu \mathrm{m}^{2}$ fields of view there were 4 and $2.56 \mu \mathrm{m}$ overscans, respectively, in the fast scan direction. The overscan must be taken into account in this study due to the manipulation taking place along the entire fast scan trace. As a result the imaged areas are quoted as $24 \times 20 \mu \mathrm{m}^{2}$ and $12.56 \times 10 \mu \mathrm{m}^{2}$. The outcome was then imaged over a larger field of view with a soft lever. The manipulation and imaging was carried out in the constant lever deflection mode where the loading forces described are the initial loading forces as the probe comes into contact with the surface and establishes feedback conditions, that is, snaps into contact and establishes an equilibrium prior to the commencement of scanning. Imaging of the manipulated regions on the polymer was carried out immediately after alteration (ca. 5 minutes) of the surface unless otherwise stated. Subsequent imaging after 1 week showed no discernable changes to the topography.

Figure 1 shows a basic schematic representation defining the directions of travel which constitute a full raster cycle. The slow scan direction of tip travel is parallel to the long axis of the lever (along the $\mathrm{x}$ direction).The fast scan direction is orthogonal to the long axis of the lever, that is, along the y direction).

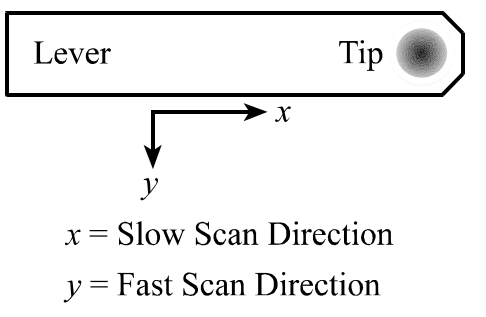

Figure 1. Schematic representation defining the probe orientation in relation to the slow (x) and fast (y) scan directions.

\section{Results and Discussion}

\subsection{Surface topography and tip indentation}

Figure 2(a) shows a contact mode topographical image of a freshly spun PDMS surface in 3-dimensional representation. The image was obtained using a soft lever (probe 'A' defined in table 2) in order to evaluate the 'true' polymer surface topography with minimal surface alteration (initial loading force of < $20 \mathrm{nN}$ ). This was confirmed by repetitive scanning over the same region and imaging conditions, with minimal resolvable changes in topography, and also by scanning a larger field of view including the previously imaged regions. The resultant image showed no resolvable change in surface characteristics/relief and no build up of displaced material at the scan edges. The surface roughness for a $1 \times 1 \mu \mathrm{m}^{2}$ region was $0.5 \pm 0.1 \mathrm{~nm}$. 

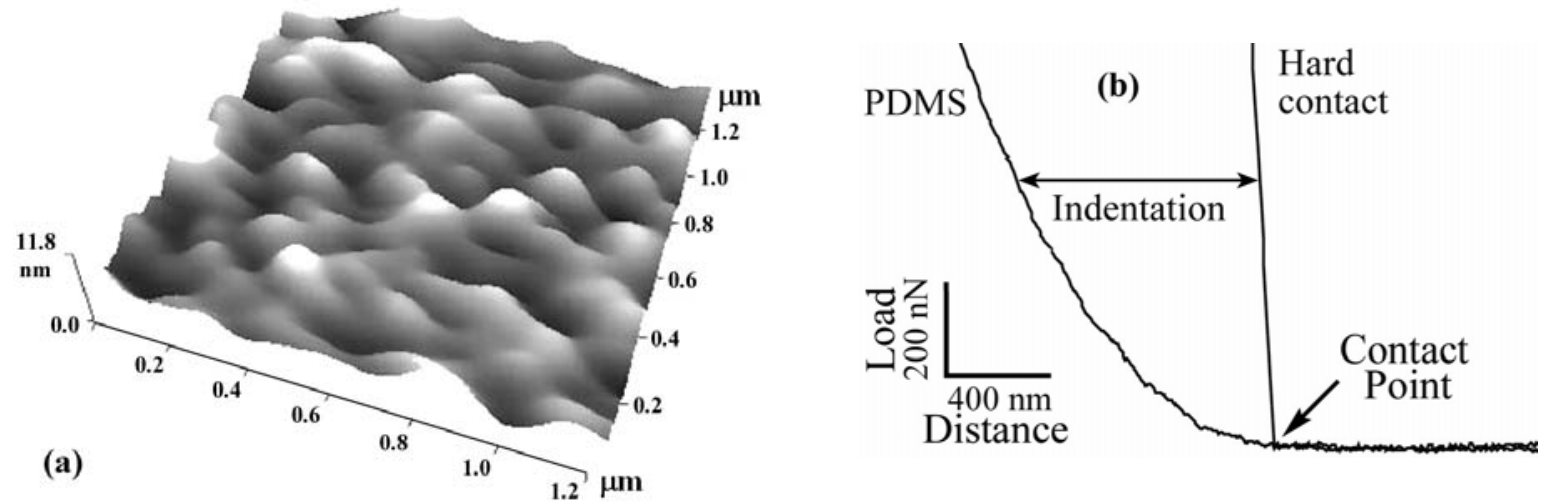

Figure 2. (a) Surface topography of a freshly spun PDMS surface shown in 3-D format. The contact mode image was obtained with probe 'A' at a constant lever deflection of $<20 \mathrm{nN}$. (b) Representative f-d curves obtained with probe 'B' for an incompressible surface, polished Si, and a PDMS surface. Tip penetration/indentation of ca. $950 \mathrm{~nm}$ is evident for an imposed force of ca. $600 \mathrm{nN}$. Subsequent imaging of the field of view after $\mathrm{f}$-d analysis revealed total elastic recovery, within the resolution of imaging.

Figure 2(b) shows the approach half-cycles of two representative force versus distance (f-d) curves obtained on a 'hard' surface (clean silicon wafer) and a PDMS surface. Note: The contact point for both curves has been aligned. The f-d data were obtained with probe 'B' (with a spring constant approximately 200 hundred times greater than probe 'A'). The shape of the f-d curve taken on the PDMS surface shows significant out-of-plane deformation of the polymer. At a force of $600 \mathrm{nN}$, there was an indentation, $\Delta z$, of ca. $950 \mathrm{~nm}$ of the PDMS surface. Using a model by Sneddon for deformation by a conical tip (see equations 1 and 2 below) as a function of sample indentation (Sneddon 1965), Young's Modulus for the PDMS material was determined to be $7.6 \times 10^{5} \mathrm{~Pa}$. The result is in good agreement with the values quoted in the literature, e.g., $7.5-17 \times 10^{5}$ Pa (Armani et al 1998, McDonald and Whitesides 2002, RocaCusachs et al 2005). Incomplete elastic recovery and/or plastic deformation arising from the indentation, in combination with the approximation of the probe being a conical indenter, may account for the slight variations in the Young's Modulus values. Adhesive forces were minimal, (in most cases the lift-off feature was not measurable), in comparison with the high values of constant lever deflections used in the manipulation experiments.

$$
F_{\text {cone }}(\Delta z)=\frac{2 E^{*}}{\pi \tan \alpha} \Delta z^{2}
$$

where $F$ is the total force (lever deflection), $\alpha$ is the half angle of a conical tip, $\Delta z$ is the indentation, and $E^{*}$ is the relative Young's modulus defined in equation 2

$$
\frac{1}{E^{*}}=\frac{1-\mu_{\text {tip }}^{2}}{E_{\text {tip }}}+\frac{1-\mu_{\text {sample }}^{2}}{E_{\text {sample }}}\left(\approx \frac{1-\mu^{2}}{E_{\text {sample }}} \text { for } E_{\text {tip }}>>E_{\text {sample }}\right)
$$


where $\mu$ is Poisson's ratio (ratio of transverse to longitudinal strain of the material under stress).

\subsection{Stick-slip in the slow scan direction}

A freshly cured PDMS sample was raster scanned using the Discoverer instrument over a $24 \times 20 \mu \mathrm{m}^{2}$ field of view, using a lever with a spring constant, $k_{N}$, of ca. $2 \mathrm{Nm}^{-1}$ (probe C in Table 2). Figure 3(a) and (b) shows the resultant PDMS surface after manipulation (contact mode imaging in a raster pattern). At relatively low force loadings $(<100 \mathrm{nN})$ the outcome of the manipulation process shows a region exhibiting higher friction in relation to the surrounding unmodified polymer surface (when imaged using a very soft lever $\left(k_{N}<<0.05 \mathrm{Nm}^{-1}\right)$ in order to minimise any further manipulation). A similar effect has been observed on other polymer surfaces (e.g., Haugstad et al 1998, Schmidt et al 2003a). Interestingly, at higher forces (> $400 \mathrm{nN}$ ) an entirely different manipulation outcome was observed (see figure 3 (c)). The image shows a series of uniformly spaced lines. The two distinctly different outcomes shown in figure 3 (b) and (c) demonstrate the ability to form homogeneous and inhomogeneous frictional surface profiles using manipulative atomic force microscopy. Figure 3 (d) shows the topographical outcome revealing the result of the manipulation process where a series of equally spaced channels is formed on the surface.
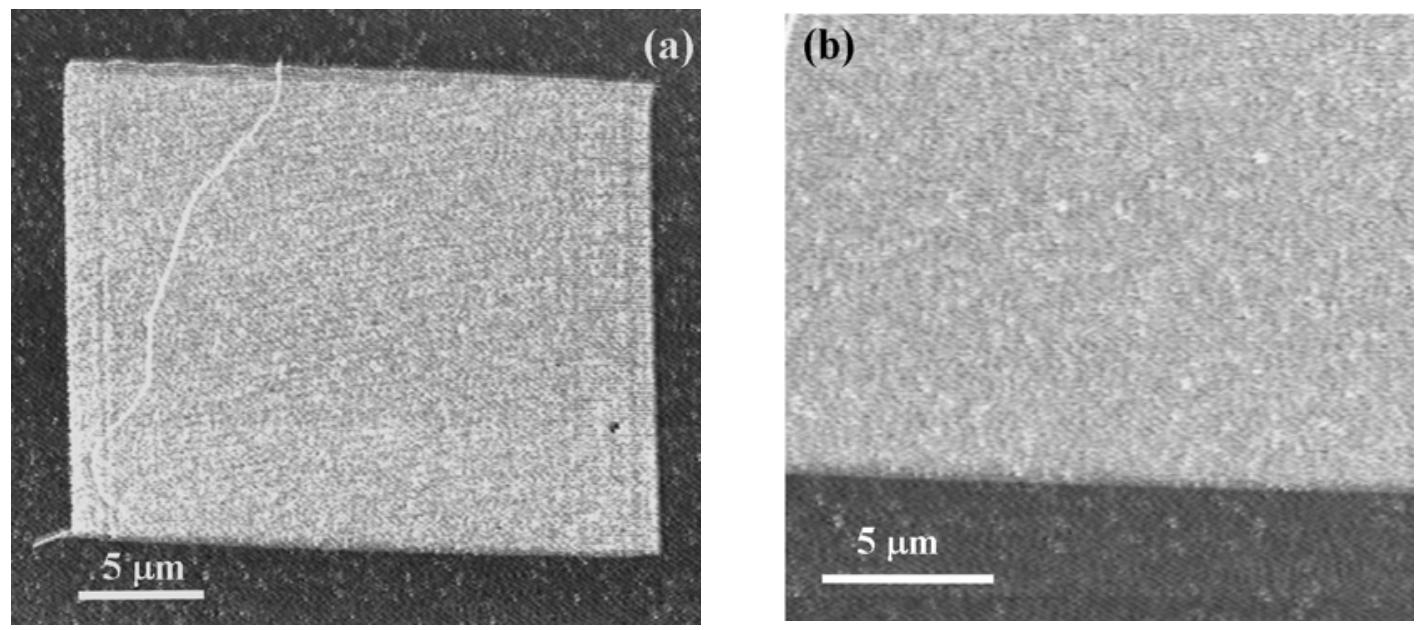

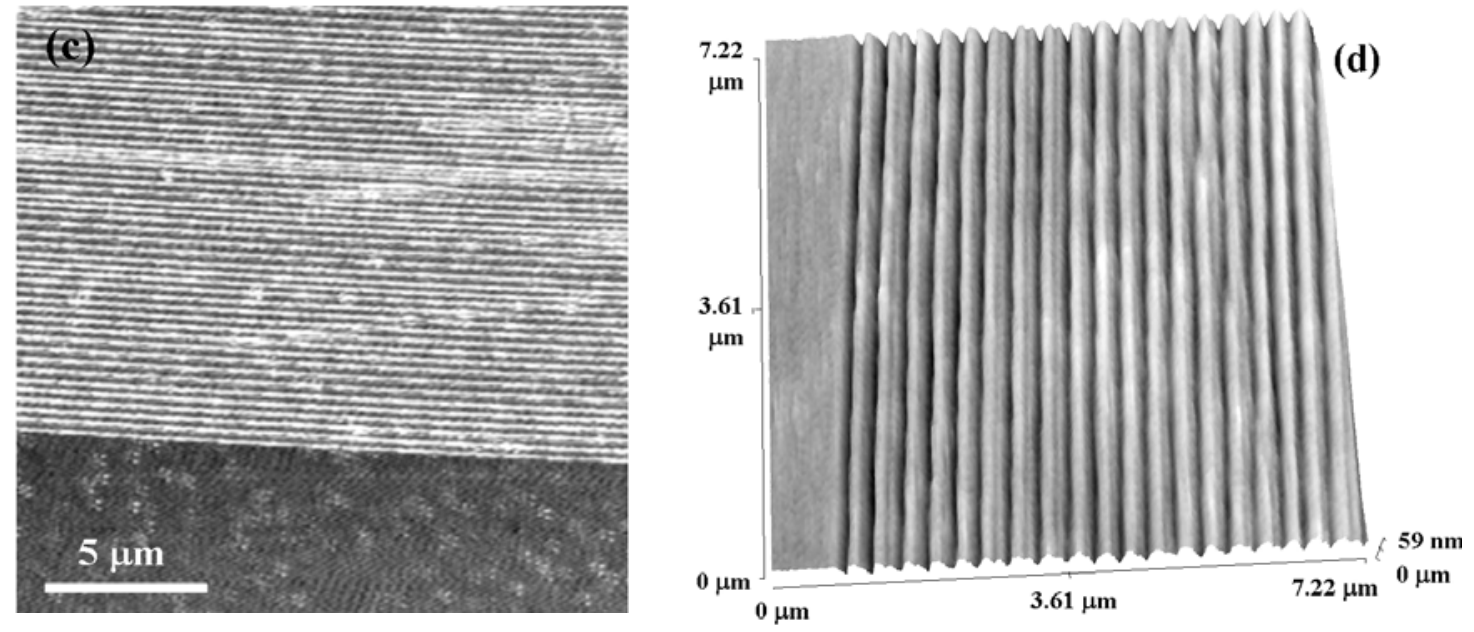

Figure 3. (a) Frictional force image of a previously manipulated region (manipulation force ca $100 \mathrm{nN}$ ). The image shows a uniform, laterally differentiated region, with the brighter square region indicating a higher frictional force. (b) A close-up of the frictional force image shown in figure (a) revealing in closer detail the uniformity of the manipulated region. (c) Frictional force image of a previously manipulated region (manipulation force ca. $400 \mathrm{nN}$ ) showing uniform lines created. (d) A 3-dimensional topographical image revealing the uniformly spaced channels formed as a result of the manipulation process.

The PDMS surface was rastered under conditions of moderately high (> $500 \mathrm{nN}$ ) force loadings in order to investigate the process of manipulation in greater detail. The field of view was generated by 600 scan lines (forward + reverse). The velocity in the fast scan direction was $125 \mu \mathrm{ms}^{-1}$, and the raster over a field of $24 \times 20 \mu \mathrm{m}^{2}$ was carried out in the constant lever deflection (with probe 'D'). The applied normal force was $950 \mathrm{nN}$ and the fast scan direction $(x$-axis, $24 \mu \mathrm{m}$ ) was perpendicular to the long axis of the lever (see inset in figure $4(\mathrm{a}))$.

Contact and lateral force images of the scanned area were then obtained with a probe (' $\mathrm{E}$ ' in table 2) having a force constant approximately 2 orders of magnitude less than that being used to manipulate the region, thus minimizing any further tip-induced alteration of the surface. The topographical image in figure 4(a) shows a series of channels created during the manipulation cycle, while the lateral force image provides enhanced contrast and resolution due to the torsional response of the lever to in-plane forces arising from changes in slope and contact area. The series of similarly spaced discontinuities/channels along the slow scan direction shows that stick/slip is taking place during the manipulation process. Figure 4(b) showing the lateral force image, reveals the stick locations (the horizontal lines) and slip lines joining the stick regions. The discontinuities/channels have an average spacing of approximately $2.3 \mu \mathrm{m}$. The location in the slow scan direction is incremented every two scanning lines in the fast scan direction in order to generate the raster pattern (see inset in figure 4(d)). Thus the scanning stage movement for a 20 
$\mu \mathrm{m}$ image (slow scan direction) incorporates $300 \times 2$ scan lines spaced $66.67 \mathrm{~nm}$ apart. Thus there will be ca. 69 scan lines for a $2.3 \mu \mathrm{m}$ stage-path along the slow scan direction, equal to the number of traverses per channel.
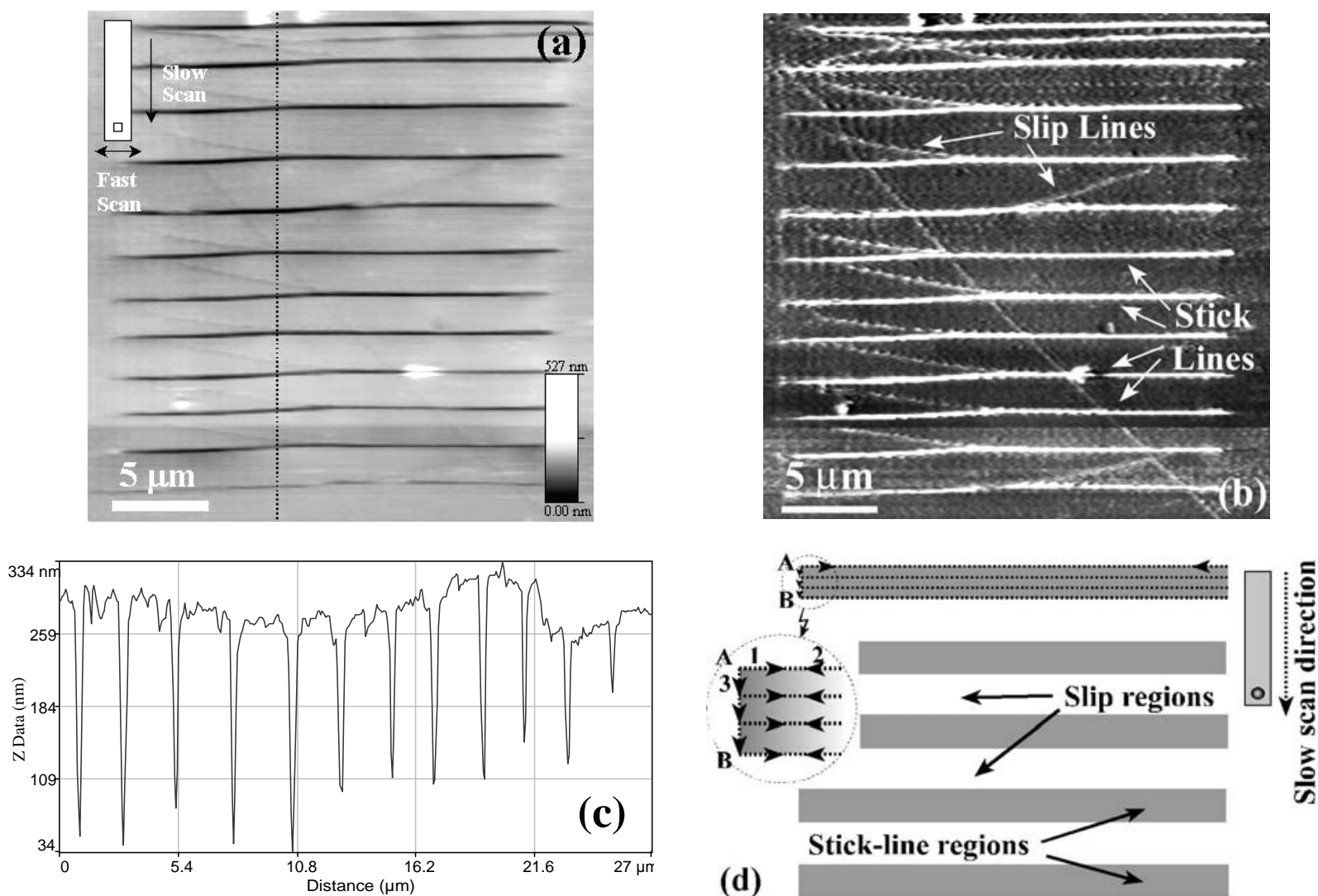

Figure 4. (a) Topographical and (b) a lateral force image of the manipulated region carried out with a soft lever (probe 'E', table 2). The resulting stick lines and the path taken by the tip during the slip stage (arrows) are evident. A contour line along the slow scan direction is shown in (c). (d) shows a diagrammatic representation of the tip sticking, A to B (raster motion from 1 to 3 within a single gray band (channel) shown in the inset), then slipping through to the next region. Tip orientation is shown in the inset in (a).

The line profile along the slow scan direction (in figure 4(c)), shows that the spacing, width and depth of the channels were in the range 1.9-2.1 $\mu \mathrm{m}, 0.4-0.6 \mu \mathrm{m}$ and $160-260 \mathrm{~nm}$, respectively. Since the timedependent position of the tip is quasi-static in comparison with any mechanical relaxation time of the polymer, it is clear that elastic recovery is taking place (the f-d data in figure 1(b) show that there is an indentation of ca. $800 \mathrm{~nm}$ at comparable force loadings).

At this point it is useful to compare tip motion during raster scanning on the PDMS with that for a 'standard' sample, that is, a hard incompressible surface. In the case of a hard surface e.g., silicon, 
movement of the stage in the fast or slow scan direction are mirrored by uniform tracking by the tip apex on the sample surface (for large stage movements of 10's of $\mathrm{nm}$ ). The situation is different in the case of the PDMS surface, where the tip is being elastically restrained at a stick point/region. When lateral restraining forces exceed that of the opposing lever-imposed force, the tip will be confined to a stick region. From figure 4 (a) and (b), it can be seen that a movement of the stage of approximately $1.8 \mu \mathrm{m}$ is required before the tip is released to the new equilibrium position. Thus at the start of the raster, the tip travels across the surface of the polymer in the fast scan direction and will presumably cause some polymer bond breakage, as well as plastic and elastic deformations. The tip remains in the channel during successive scans and displaces more polymeric material as the scanning continues, while being deflected in the direction of the slow scan, until a lateral force causes escape from the stick-region, and allows the next point of tip-polymer stability to be established. That is, the effective spring constant of lateral interaction exceeds the effective in-plane spring constant of the lever, when the tip is trapped, while the reverse is the case when it escapes in a discontinuous manner. Figure 4(d) shows a diagrammatic representation of the manipulation event, where the tip begins its raster motion at point A moving towards point B (as illustrated in the inset whereby the raster motion proceeds from 1 to 3 within a single gray band (channel)), becoming 'stuck’ within the broad horizontal bands representing one full stick cycle. As a consequence, only a small spatial region along the slow scan direction is imaged. As there will be significant polymer deformation during this process the stick region along the slow scan direction will be dragged along due to the longitudinal/buckling force imposed by the lever and displaced to the tip polymer contact region.

\subsection{Stick-slip in the fast scan direction}

In-plane forces acting along the long axis of the lever (giving rise to buckling) and 'normal' (bending) forces are both detected on the top-bottom segments of the photo-detector; thus it is difficult to decouple experimentally the contributions of these deformational modes of the lever along the slow scan direction. However, the left-right segments of the photo-detector measure lateral forces imposed orthogonal to both the normal lever deflection forces and the long axis of the lever. The lateral twisting forces along the fast scan direction can therefore be monitored simultaneously and unambiguously with minimal cross-talk between the two signals.

Five representative friction loops are shown in figure 5 representing the lateral forces acting on the tip in the fast-scan direction (probe ' $F$ ') during the manipulation process, thus revealing the lateral response of the lever in real space and time. The lateral force sensed by the tip is plotted along the vertical scale of the friction loops ( $F_{L}$ in figure 5) while the stage displacement in the fast scan direction is plotted along the horizontal scale. The lateral force responses of the lever were obtained while scanning (in a raster pattern) 
with a loading force of $1100 \mathrm{nN}$. For comparison a friction loop on a clean silicon surface is shown in figure 5(a). The inset in figure 5(a) shows the near-vertical slope in the static friction region that is characteristic of a hard non-compliant surface such as Si. The friction coefficient on the Si surface was $0.1 \pm 0.05$, which is in close agreement with values in the literature (e.g., $0.09 \pm 0.03$ (Crossley et al., 1998)).

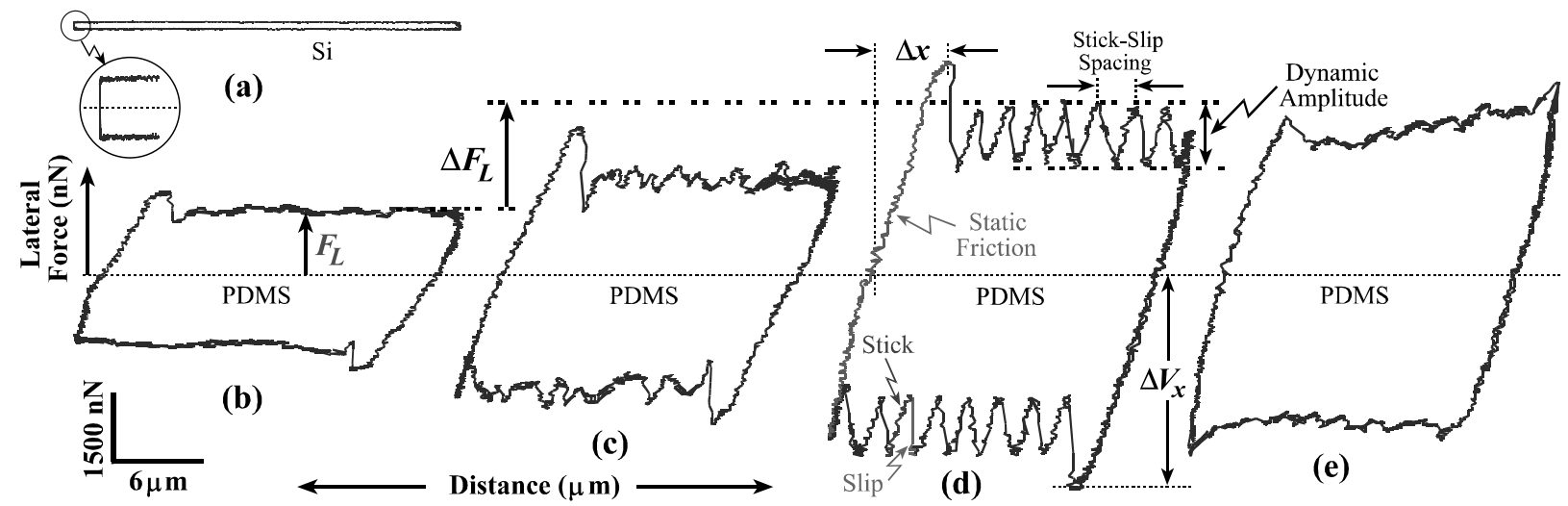

Figure 5. (a) Calibration friction loop obtained on a silicon surface with the inset showing the detail of the vertical slope. In (b), (c), (d) and (e) are shown friction loops obtained on a PDMS surface (probe 'F', table 2) illustrating the progression of the stick-slip behaviour in both the $x$ and $y$ directions. The loop in (b) represents the beginning of a cycle with an increase in lateral force in both the $x$ and $y$ scan directions, progressing to the loop in $(c)$ and finally to $(d)$ which clearly shows the characteristic stick-slip features. A few rasters prior to the tip slipping (e), the stick-slip features degrade completely. $\Delta V_{x}$ in (d) represents the L-R detector signal with $\Delta x$ showing the lateral (in-plane) displacement of the stage.

Figure 5(b)-(e) show progressive friction loops taken on the PDMS sample while the tip is confined to one particular trough during the manipulation cycle. The lateral force exhibited a progressive increase, from ca. 1200 to ca. $2500 \mathrm{nN}$, while the tip remained trapped within a channel. Thus the lateral response in the case of the PDMS surface is markedly different to that of a silicon surface. During the first few traverses the lateral force response was 'flat' (figure 5(b)), with minimal changes of magnitude in the region of dynamic friction. However, stick-slip behaviour became increasingly prominent, as seen in figure 5(c) with the periodicity and amplitude in the dynamic region of the stick-slip responses in figure 5(d) becoming highly regular. Finally, during the last few traverses before the tip escapes from the channel, the stick-slip pattern gradually becomes less distinct (figure 5(e)).

Figure 6 shows a schematic representation of the stick-slip behaviour in the fast scan direction similar to that shown earlier for the stick-slip process in the slow scan direction (figure 4(d)). As the tip moves across the surface ( $x$-direction) it becomes trapped at the first stick point until the torsional force of the tip 
exceeds the stick force; the tip then is released and jumps to its next stick position, repeating the cycle in both the forward and reverse directions of travel.

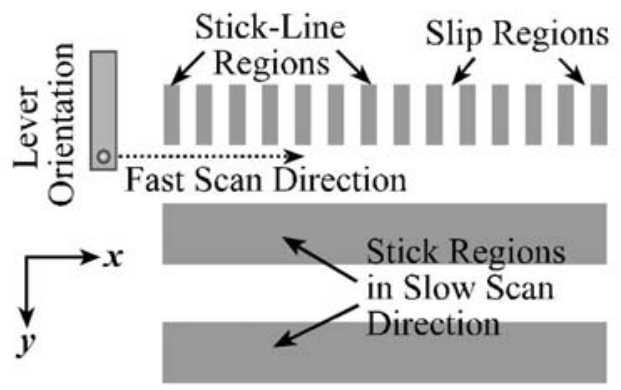

Figure 6. Schematic representation of the fast scan stick-slip motion of the lever. The grey bands represent a stick region in both the fast and slow scan directions. The lever is oriented orthogonally to the fast scan direction.

The repeat distance of stick-slip features in the fast scan direction is ca. $2.3 \mu \mathrm{m}$, figure $5(\mathrm{~d})$. This is similar to the distances observed in the spacing between channels generated in the slow scan direction at similar 'normal' force loadings, figure 4(b). The lateral force response of the probe ('F' in table 2) along the two directions can be attributed to the corresponding force constants $k_{T}\left(220 \mathrm{Nm}^{-1}\right)$ and $k_{L}\left(145 \mathrm{Nm}^{-1}\right)$, which are similar in magnitude.

\subsection{Stick-slip dependence on the initial loading force}

Figure 7 shows representative friction loops taken at the same stage of the evolution of a channel (corresponding to the position as defined in figure 5(d)), and with the same probe (' $\mathrm{H}$ '). These show the extent of in-plane displacement $(\Delta x)$ of the stick-point as the lever deflection is increased. Segments (1) to (4) show the extent of increase in sample displacement with an increasing loading force. These were; (a) $1150 \mathrm{nN}$, (b) $2600 \mathrm{nN}$, (c) $4100 \mathrm{nN}$, and (d) $5550 \mathrm{nN}$, with the corresponding in-plane displacement of the polymer being (1) $4.3 \mu \mathrm{m}$, (2) $6 \mu \mathrm{m}$, (3) $7 \mu \mathrm{m}$, and (4) $9.7 \mu \mathrm{m}$. It is also apparent that as the loading force was increased, the repeat spacing between stick-slip features increased, reflecting an increase in trapping efficiency with normal force. The results of altering the loading force, scan speed and image resolution will be explored further in a subsequent publication. 


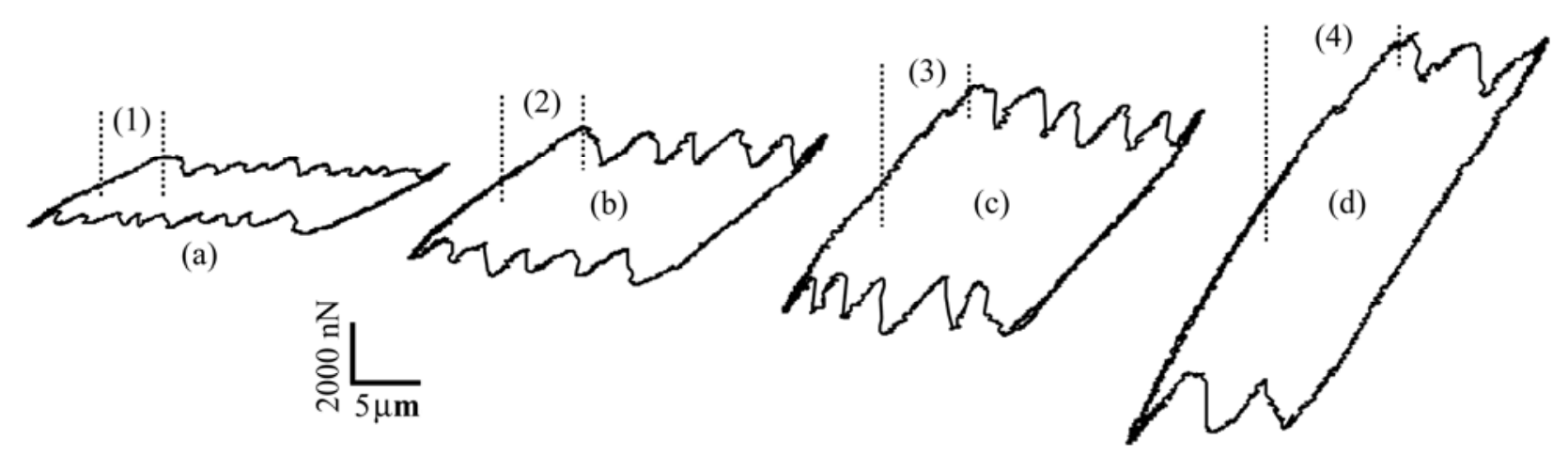

Figure 7. Representative friction loops (middle of the cycle) using the same probe (probe ' $\mathrm{H}$ ', table 2) but varying the loading force; (a) $1150 \mathrm{nN}$, (b) $2600 \mathrm{nN}$, (c) $4100 \mathrm{nN}$, and (d) $5550 \mathrm{nN}$. Segments (1) to (4) show the in-plane displacements of the stick points; 4.3, 6, 7, $9.7 \mu \mathrm{m}$, respectively.

The friction loop data presented in figure 7 were plotted graphically (see figure 8) and revealed a linear dependence of the in-plane displacement on loading force.

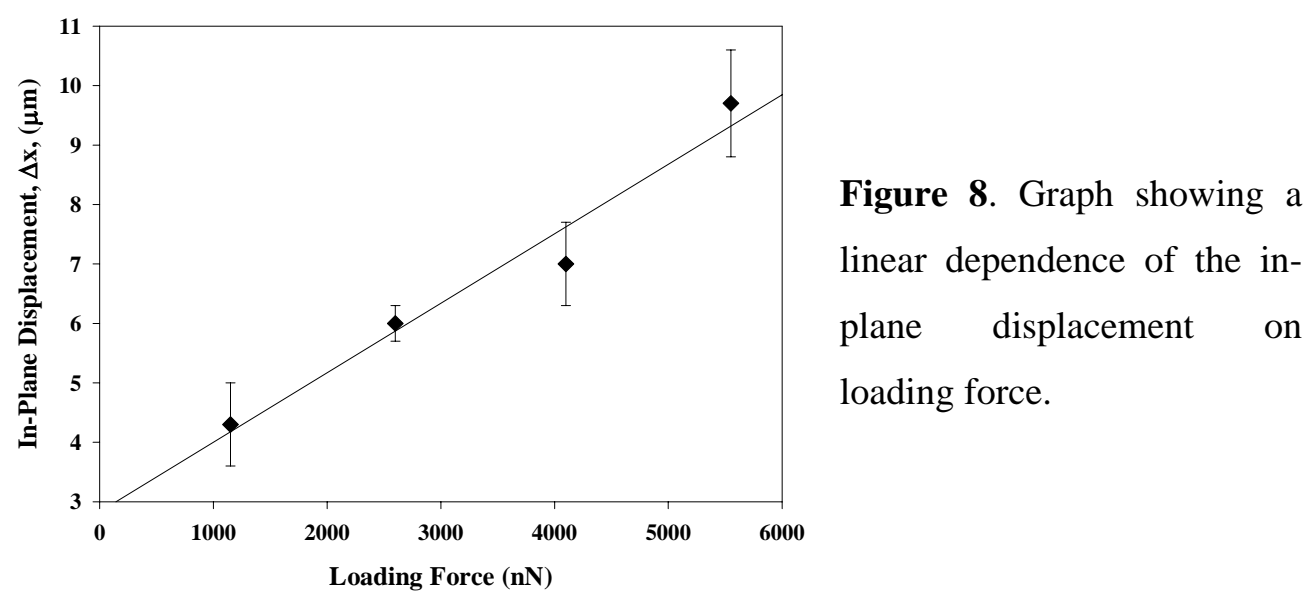

Figure 9(a) shows the general trend whereby the systematic increase in lever deflection (loading force) results in greater 'dynamic' stick-slip amplitude (probe ' $\mathrm{B}$ '); the term 'dynamic' refers to stick-slip in the fast scan direction and is defined in figure 5(d). The dependence was linear. In essence the result says that forces corresponding to release from, and trapping at, a stick point is linearly dependent on the lever deflection (loading) force. The repeat distance between the stick-slip features is shown in figure 9(b). The graphs demonstrate an increase in the repeat distance with increasing lever deflection (loading force). Again, the dependence appears linear, and shows that in-plane polymer deformation is linearly dependent on lever deflection (loading) force. The results reflect the trapping efficiency of the polymer-to-tip contact as a function of load, and are linearly related to the deformation of the polymer. The deformation of the polymer and the efficiency of the stick-point are clearly related to indentation by the tip. 

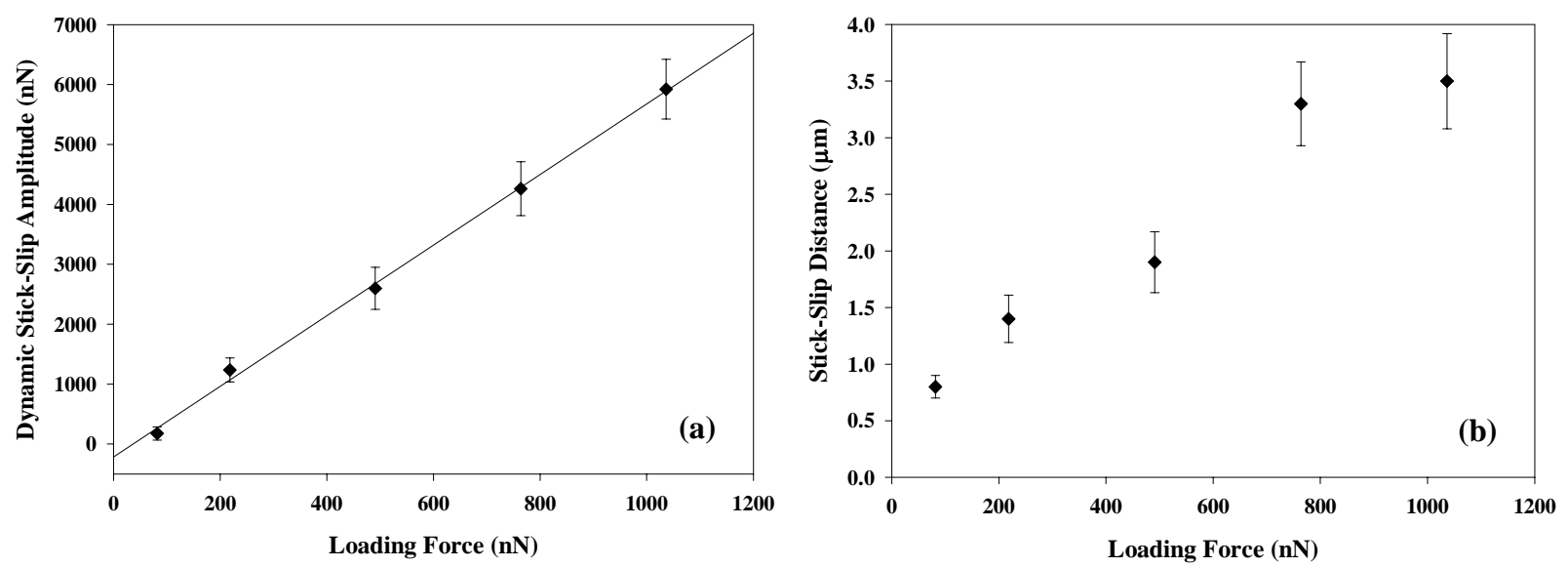

Figure 9. Data for fast scan direction. (a) The dependence of stick-slip amplitude on lever deflection (initial loading force). (b) The dependence on lever deflection (initial loading force) of repeat distance of stick-slip in the fast scan direction. Data were obtained with probe 'B'.

Similar results were obtained from investigation of the dependence on load of stick-slip in the slow scan direction, illustrated by post-manipulation imaging. The manipulation was carried out with probe ' $\mathrm{F}$ ' over an area of $24 \times 20 \mu \mathrm{m}^{2}$, where the loading force was decreased during the raster. Figure 10 shows the resulting topographical image (obtained with probe ' $G$ '). The image shows an increase in the number of discontinuities per unit stage travel with decreasing load. The channel length was found to increase with decreasing load in accord with data from friction loop analysis (see figures 7 and 8). This effect can be attributed to the increase in static friction with normal force, causing greater surface deformation, and thus a shorter channel length.

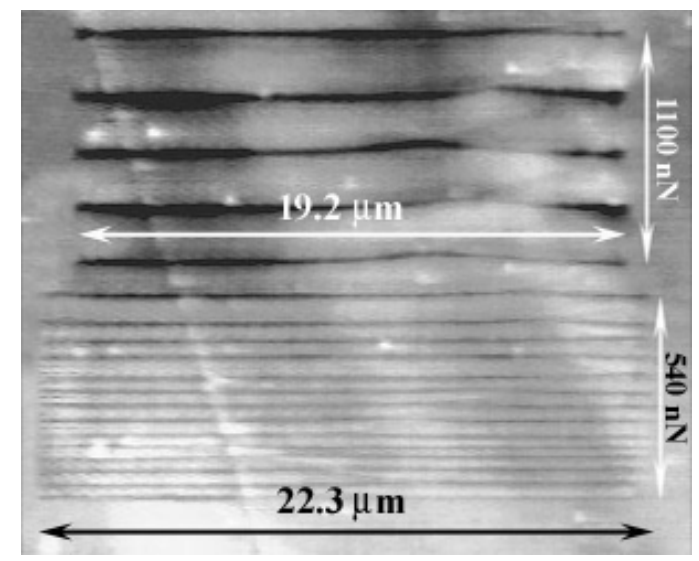

Figure 10. Topographical image of the manipulated region obtained with a low spring constant lever (probe ' $G$ ', table 2) showing an increase in channel length, for the same scanner excursion, with a decrease in loading force. A decrease of the in-plane deformation of the polymer is also evident. A dependence of channel spacing on load is apparent.

\section{Conclusion}

Stick-slip effects have been observed in previous AFM studies; these have, in most cases, been restricted to atomic and molecular scales (Watson et al 2004, Mate et al 1987, van den Oetelaar et al 1997). In the 
case of AFM-based manipulation of polymers the focus has generally been on establishing the dependence on loading force (Blach et al 2004, Garnaes et al 1994, Ling et al 1998, Fang et al 2000, Blach et al 2004), and/or temperature and number of rasters (Schmidt et al 1999, 2003a, 2003b). The present results describe the response of the AFM probe when it is in dynamic contact with a soft elastic polymer surface by analysis of frictional loop data and lithographic outcomes.

Surface alteration has been correlated with the response of the probe to linear motion and to lateral forces imposed by the relaxing polymer. An explanatory model was constructed whereby the tip not only sticks and slips within the channel in the fast scan direction, but also from one channel to another, in the slow scan direction. The process was consistently reproduced with levers with a spring constant of $>1 \mathrm{Nm}^{-1}$. The paper also demonstrates the control of lithographic outcomes (patterning) e.g., channel spacing. It may be possible to incorporate this patterning process using an array of levers as opposed to a single beam.

\section{Acknowledgements}

J. A. Watson wishes to acknowledge funding from the Cooperative Research Centre for MicroTechnology. The authors would also like to thank Ray Sweatman for his assistance with PDMS spin-coating.

\section{References}

Please note a formal change of name from J A Blach to J A Watson in June 2004.

Armani D, Liu C and Aluru N 1998 12 ${ }^{\text {th }}$ Int. Conf. on MEMS (Orlando Florida) p 222

Blach J A, Watson G S, Brown C L, Pham D K, Wright J, Nicolau D V and Myhra S 2004 A mechanistic approach to tip-induced nano-lithography of polymer surfaces Thin Solid Films 459 95-99

Blach-Watson J A, Watson G S, Brown C L and Myhra S 2004 UV patterning of polyimide: differentiation and characterization of surface chemistry and structure Applied Surface Science 235, 164-169

Bhushan B 2001 Nano- to microscale wear and mechanical characterization using scanning probe microscopy Wear 251 1105-1123

Brinkmann M, Chan V Z-H, Homas E L, Lee V Y, Miller R D, Hadjichristidis N and Avgeropoulos A 2001 Room-temperature synthesis of $a-\mathrm{SiO}_{2}$ thin films by UV-assisted ozonolysis of a polymer precursor Chemical Materials 13, 967- 972

Chaudhury M K and Whitesides G M 1991 Direct measurements of interfacial interactions between semispherical lenses and flat sheets of Poly(dimethylsiloxane) and their chemical derivatives Langmuir 7 1013-1025 
Cleveland J P, Manne S, Bocek D and Hansma P K 1993 A nondestructive method for determining the spring constant of cantilevers for scanning force microscopy Review of Scientific Instruments 64 403-405

Crossley A, Johnston C, Watson G S and Myhra S 1998 Tribology of diamond-like-carbon films from generic fabrication routes investigated by lateral force microscopy Journal of Physics D: Applied Physics 31 1955-1962

Deng T, Prentiss M and Whitesides G M 2002 Fabrication of magnetic microfiltration systems using soft lithography Applied Physics Letters 80 461-463

Dow Corning Corp., Sylgard-184, Midland, MI, http://www.dowcorning.com.

Fang T-H, Went C-I and Chang J-G 2000 Machining characterization of the nano-lithography process using atomic force microscopy Nanotechnology 11 181-187

Elkaakour Z, Aime J P, Bouhacina T, Odin C and Masuda T 1994 Bundle formation of polymers with atomic force microscope in contact mode: A friction versus peeling process Physical Review Letters 73 3231-3234

Garnaes J, Bjornholm T and Zasadzinski J A N 1994 Nanoscale lithography on langmuir-blodgett films of behinic acid Journal of Vacuum Science and Technology B 12 1839-1842

Gibson C T, Watson G S and Myhra S 1997 Scanning force microscopy - calibration procedures for 'best practice’ Scanning 19 564-581

Haugstad G, Gladfelter W L and Jones R R 1998 Scanning force microscopy characterization of viscoelastic deformations induced by precontact attraction in a low cross-link density gelatin film Langmuir 14 3944-3953

Hector L G and Schmid S R 1998 Simulation of asperity plowing in an atomic force microscope Part I: Experimental and theoretical methods Wear 215 247-256

Hillborg H and Gedde U W 1999 Hydrophobicity changes in silicone rubbers, IEEE Transactions on Dielectric and Electrical Insulation 6 703-717

Hu J, Deng T Beck R G, Westervelt R M and Whitesides G M 1999 Fabrication of arrays of Schottky diodes using microtransfer molding Sensors \& Actuators A 75 65-69

Ikada Y 1994 Surface modification of polymers for medical applications Biomaterials 15 725-736

Kane R S, Takayama S, Ostuni E, Ingber D E and Whitesides G M 1999 Patterning proteins and cells using soft lithography Biomaterials 20 2363-2376

Kim J, Chaudhury M K and Owen M J 1999 Hydrophobicity loss and recovery of silicone HV insulation IEEE Transactions on Dielectric and Electrical Insulation 6 695-702

Ling J S G, Leggett G J and Murray A J 1998 Scanning force microscopy of polyester films: Contact versus non-contact imaging and tip-induced wear experiments Polymer 39 5913-5921 
McDonald J C and Whitesides G M 2002 Poly(dimethylsiloxane) as a material for fabrication microfluidic devices Accounts of Chemical Research 35 491-499

Mate C M, McClelland G M, Erlandsson R and Chiang S 1987 Atomic-scale friction of a tungsten tip on a graphite surface Physical Review Letters 59 1942-1945

Nicolau D V, Taguchi T, Taniguchi H and Yoshikawa S 1999 Negative and positive tone protein patterning on E-beam/deep-UV resists Langmuir 15 3845-3851

Olah A, Hillborg H and Vancso G J 2005 Hydrophobic recovery of UV/ozone treated poly(dimethylsiloxane): adhesion studies by contact mechanics and mechanism of surface modification Applied Surface Science 239 410-423

Pickering J P and Vancso G J 1999 On the formation of oriented nanometer scale patterns on amorphous polymer surfaces studied by atomic force microscopy Applied Surface Science 148 147-154

Pike J K, Ho T and Wynne K 1996 Water-induced surface rearrangements of Poly(dimethylsiloxane-ureaurethane) segmented block copolymers Chemistry of Materials 8 856-860

Roca-Cusachs P, Rico F, Martinez E, Toset J, Farre R and Navajas D 2005 Stability of microfabricated high aspect ratio structures in Poly(dimethylsiloxane) Langmuir 21 5542-5548

Schmid S R and Hector L G 1998 Simulation of asperity plowing in an atomic force microscope. Part II: Plowing of aluminium Alloys Wear 215 257-266

Schmidt R H, Haugstad G and Gladfelter W L 1999 Correlation of Nanowear patterns to viscoelastic response in a thin polystyrene melt Langmuir 15 317-321

Schmidt R H, Haugstad G and Gladfelter W L 2003a Scan-induced patterning in glassy polymer films: Using scanning force microscopy to study plastic deformation at the nanometer length scale Langmuir 19 898-909

Schmidt R H, Haugstad G and Gladfelter W L 2003b Scan-induced patterning and the glass transition in polymer films: Temperature and rate dependence of plastic deformation at the nanometer length scale Langmuir 19 10390-10398

Schnyder B, Lippert T, Kotz R, Wokaun A, Graubner V-M and Nuyken O 2003 UV-irradiation induced modification of PDMS films investigated by XPS and Spectroscopic Ellipsometry, Surface Science 532-535 1067-1071

Schueller O J A, Brittain S T and Whitesides G M 1999 Fabrication of glassy carbon microstructures by soft lithography Sensors \& Actuators A 72 125-139

Singer I L 1994 Friction and energy dissipation at the atomic scale: A review J. Vac. Sci. Technol. A 12 2605-2616

Sneddon N 1965 The relation between load and penetration in the axisymmetric boussinesq problem for a punch of arbitrary profile International Journal of Engineering Science 3 47-57 
Unger M A, Chou J-P, Thorsen T, Scherer A and Quake S R 2000 Monolithic microfabricated valves and pumps by multilayer soft lithography Science 288 113-116

van den Oetelaar R J A and Flipse C F J 1997 Atomic-scale friction on diamond (111) studied by ultrahigh vacuum atomic force microscopy Surface Science Letters 384 L828-L835

Watson G S, Dinte B P, Blach-Watson J A and Myhra S 2004 Friction measurements using force versus distance friction loops in force microscopy Applied Surface Science 235 38-42

Xia Y and Whitesides G M 1998 Soft lithography Angewandte Chemie International Edition 37 550-575 